\title{
50 Millionen Gutmenschen für gesetzlichen Mindestlohn in Deutschland
}

Ronald Schettkat

Rund $70 \%$ der Bundesbürger sind laut einer Umfrage der ZEIT für die Einführung eines Mindestlohnes, was sicher auf die spürbare Zunahme der Lohnspreizung in Deutschland zurückzuführen ist. Befürwortern gesetzlicher Mindestlöhne wird häufig eine moralisch honorige Intention, aber zugleich auch fehlender Realitätsbezug unterstellt. Gutmenschen eben. Wer die Flexibilität der Löhne nach unten begrenzt, der vernichte Beschäftigungsmöglichkeiten, insbesondere für geringer qualifizierte Arbeitnehmer, doziert der Sachverständigenrat.

Aber warum fordern dann in den USA sogar Nobelpreisträger der Wirtschaftswissenschaften - unter ihnen wahre Giganten der Profession wie Kenneth Arrow (Stanford) und Robert Solow (MIT) - eine Heraufsetzung des gesetzlichen Mindestlohnes? Haben die das Einmaleins der ökonomischen Theorie nicht verstanden? Was ist mit unseren westeuropäischen Nachbarn, die entweder gesetzliche Mindestlöhne (Niederlande, Belgien, Frankreich, England), einen durch die Tarifpartner vereinbarten Mindestlohn (Österreich) oder eine nahezu $100 \%$ ige tarifliche Absicherung der Löhne haben (Skandinavien) und die teilweise zu wahren Beschäftigungswundern hochstilisiert wurden?

Theoretisch ist die negative Beschäftigungswirkung von Lohnuntergrenzen lediglich im Extremmodell des perfekten Marktes eindeutig negativ. In realistischeren Modellen werden Löhne durch Verhandlung bestimmt und sind nicht durch ein imaginäres Grenzwertprodukt determiniert, weshalb keine eindeutige Obergrenze, sondern vielmehr ein gewisser Lohnspielraum besteht. Das haben vor allem die Arbeiten von David Card und Alan Krueger gezeigt, die empirisch keine negative Beschäftigungswirkung einer immerhin fast 20 \%igen Erhöhung des Mindestlohnes feststellen konnten. Ein Ergebnis, das mit dem Modell des perfekten Marktes unvereinbar ist, weshalb die quasi-experimentellen Analysen von Card und Krueger weltweite Diskussionen und Folgeanalysen initiiert haben. Deren Ergebnisse sind widersprüchlich, bestätigen im Kern aber die Unbestimmtheit des Beschäftigungseffektes von Mindestlohnerhöhungen.

Zur Vermeidung des Lohnwettbewerbs nach unten hatte USPräsident Roosevelt 1938 einen gesetzlichen Mindestlohn eingeführt, der seither in unregelmäßigen Zeitabständen angehoben wird und deshalb zwischenzeitlich relativ zum stetig steigenden Durchschnittslohn sinkt. 1997, bei der letzten Erhöhung der US Federal Minimum Wage durch die Clinton-Administration, betrug der Mindestlohn rund $41 \%$ des durchschnittlichen Stundenlohnes von Produktionsarbeitern im privaten Sektor der US-Wirtschaft. Bis zum Jahr 2006 fiel dieser Wert auf rund $31 \%$ ab. Zur Illustration: Die vom ZEW-Präsidenten und Wirtschaftsweisen Professor Franz avisierten $3 €$ Stundenlohn entsprechen etwa $18 \%$ eines durchschnittlichen Arbeiterlohnes. Ein Hungerlohn, der in Westeuropa außerhalb der ernsthaft diskutierten Bandbreiten liegt und selbstverständlich der Subventionierung bedarf.

Natürlich wird kein Ökonom bestreiten, dass die Beschäftigungswirkung des Mindestlohnes von seiner Höhe und der davon betroffenen Arbeitnehmerzahl abhängt. Ein Mindestlohn von 4,50€ würde nach den Daten des Sozio-oekonomischen Panels (SOEP) etwa $2 \%$ der Arbeitnehmer in Westdeutschland (6\% in Ostdeutschland) betreffen, und bei 7,50€ wären es rund $11 \%$ (26\% in Ostdeutschland). Offensichtlich gibt es eine deutliche Diskrepanz in der Lohnstruktur zwischen Ost und West, weshalb man vorsichtigerweise wohl zunächst auf eine regionale Differenzierung der Mindestlöhne setzen würde. In Großbritannien hat die Blair-Regierung 1999 einen Mindestlohn - auch zur Begrenzung von Missbrauch der negativen Einkommensteuer - eingeführt, der rund $50 \%$ des Medianlohnes betrug und die Lohnungleichheit spürbar reduziert hat, aber dennoch beschäftigungsneutal war. ${ }^{1}$

Die britischen Erfahrungen kontrastieren deutlich mit den prognostizierten Beschäftigungsverlusten von 600.000 Jobs bei einem Mindestlohn von 7,50 € in Deutschland, die in einem gemeinsamen Papier des Instituts für Wirtschaftsforschung Halle und des ifo Instituts für Wirtschaftsforschung veröffentlicht wurden. Allerdings reproduzieren die Institute mit ihrer Rechnung lediglich ihre Annahme, wonach $10 \%$ Lohnerhöhung die Beschäftigung um 7,5\% reduzieren würde. Ein Wert, der weit über den empirisch ermittelten Schätzungen liegt.

Wer meint, Lohnsubventionen - durch irgendein Kombilohnmodell oder durch die Hartz IV-Mindestsicherung - könnten auf gesetzliche Mindestlöhne verzichten, ohne ein Fass ohne Boden im Lohnsenkungswettbewerb zu öffnen, der muss darauf setzen, dass Unternehmen genau das Grenzwertprodukt des Arbeitnehmers zahlen und nicht dem Reiz höherer Gewinne durch Absenkung der Löhne erliegen.

\footnotetext{
1 Metcalf, D. (2007): Why has the British National Minimum Wage had little or no impact on employment?, London School of Economics, CEP Discussion Paper 7881
}

\footnotetext{
Professor Ronald Schettkat lehrt Volkswirtschaftslehre und insbesondere Wirtschaftspolitik an der Bergischen Universität Wuppertal. Arbeitsschwerpunkte: Beschäftigungs- und Arbeitsmarkttheorie Wachstumstheorie und Makroökonomie. e-mail: schettkat@wiwi.uni-wuppertal.de
} 